\title{
SOBRE LA ANALITICIDAD DEL SEMIGRUPO CO ASOCIADO A UN SISTEMA VISCOELÁSTICO
}

\author{
Yolanda S. Santiago Ayala ${ }^{1}$
}

RESUMEN.- En este artículo, demostramos que el semigrupo $C_{0}$ asociado a un Sistema Viscoelástico es analítico y exponencialmente estable.

PALABRAS CLAVE.- Semigrupos analiticos, Semigrupos de contracción, Decaimiento exponencial, Operador resolvente, Sistema viscoelástico.

\section{ABOUT THE ANALITICITY OF THE CO-SEMPGROUP ASSOCIATED TO A VISCOELASTIC SYSTEM}

\begin{abstract}
We proved that the semigroup $C_{O}$ associated to a viscoelastic system is analytic and exponentially stable.

KEYWORDS.- Analytic semigroups, Contraction semigroups,Exponential decay, Resolvent operator, Viscoeslast system.
\end{abstract}

\section{INTRODUCCIÓN}

Estudiamos el siguiente sistema disipativo

$$
\begin{array}{r}
\left.u_{t t}+\Delta^{2} u-\Delta u_{t}=0, \text { en } \Omega \times\right] 0, T[ \\
u=\Delta u=0, \text { en } \partial \Omega \times] 0, T[ \\
u(x, 0)=u_{0}(x), u_{t}(x, 0)=u_{1}(x) \quad x \in \Omega
\end{array}
$$

donde $\Omega$ es un abierto acotado de $\mathbb{R}^{n}$ con frontera $\partial \Omega$ suave.

Este sistema modela el movimiento transversal de una placa de material viscoelástica presa en la frontera $\partial \Omega$, sin movimiento longitudinal. $u(x, t)$ representa la posición de la placa en el instante $t$ y $\Delta^{2}, \Delta, \nabla$ son los operadores Biarmónico, Laplaciano y Gradiente respectivamente.

${ }^{1}$ Profesora de la Facultad de Ciencias Matemáticas de la Universidad Nacional Mayor de San Marcos. E-mail: yssa@lycos.com 
La energía asociada al sistema es

$$
E(t):=\frac{1}{2} \int_{\Omega}\left|u_{t}\right|^{2}+|\Delta u|^{2} d x
$$

$E(t)$ es no creciente, desde que

$$
\frac{\partial E(t)}{\partial t}=-\int_{\Omega}\left|\nabla u_{t}\right|^{2} d x \leq 0
$$

Así $0 \leq E(t) \leq E(0)$

Entonces surge la interrogante ¿A dónde tiende $E(t)$ cuando $t \rightarrow \infty$ ? y ¿con qué tasa de decaimiento lo hace?.

Para abordar este punto, probaremos que el semigrupo asociado al sistema es analítico, utilizando técnicas multiplicativas y el Toerma 2 que involucra al operador resolvente.

La estabilidad exponencial se obtiene como una consecuencia del Teorema 3. Para este caso, cabe resaltar que la Teoría de Semigrupos nos garantiza la existencia, unicidad y el comportamiento asintótico de la solución.

Recordemos que un semigrupo $\mathrm{C}_{0}$ si decae, lo hace con tasa exponencial. Podemos citar un semigrupo $\mathrm{C}_{0}$ que no decae, ver Pazy [3]. Existen trabajos importantes de estabilidad via semigrupos, citamos Liu-Zheng [2] y Wyler [6].

Para caracterizaciones del espectro de un semigrupo $C_{0}$ ver Prüss [4], Adams [1], Renardy-Hrusa-Nohel [5] que son referencias bases en esta rama de la matemática.

\section{PRELIMINARES}

Usaremos las siguientes definiciones y resultados. Ver Liu - Zheng [2] y Pazy [3].

\section{Definición 1.}

Sea $H$ un espacio de Hilbert dotado de un producto interno (, ) y la norma inducida $\|\cdot\|$. Sea $A$ un operador lineal en $H$, ie. $A: D(A) \subseteq H \rightarrow H$. Diremos que $A$ es disipativo; si para cualquier $x \in D(A), \operatorname{Re}(A x, x) \leq 0$.

\section{Definición 2.}

Una familia $S(t)(0 \leq t<\infty)$ de operadores lineales acotados en un espacio de Banach $H$ es un semigrupo fuertemente continuo (un semigrupo de clase $C_{0}$ ) si satisface: 
(i ) $S\left(t_{1}+t_{2}\right)=S\left(t_{1}\right) S\left(t_{2}\right), \forall t_{1}, t_{2} \geq 0$,

(ii) $S(0)=I$,

(iii) Para cada $x \in H, S(t) x$ es continuo en $t$ en $[0, \infty)$.

Para cada semigrupo $S(t)$, definimos un operador $A$ con dominio $D(A)$

$$
D(A)=\left\{x, \exists \lim _{h \rightarrow 0} \frac{S(h) x-x}{h}\right\}
$$

entonces $A$ es llamado el generador infinitesimal del Semigrupo de $S(t)$.

\section{Definición 3.}

Diremos que $S(t)$ es exponencialmente estable si existen constantes positivas $\alpha$ y $M \geq 1$ tales que

$$
\|S(t)\| \leq M e^{-\alpha t}, \quad \forall t \geq 0
$$

\section{Definición 4.}

Diremos que $S(t)$ es analítico, si admite una extensión $T(\lambda)$ para $\lambda \in \Delta_{\theta}=\{\lambda \in \mathbb{C},|\arg \lambda|<\theta\}$ para algún $\theta>0$; tal que $\lambda \rightarrow T(\lambda)$ es analítico y satisface:

$$
\begin{gathered}
\lim _{\Delta_{\theta} \ni \lambda \rightarrow 0}\|T(\lambda) z-z\|=0 \quad, \quad \forall z \in H \\
T(\lambda+\mu)=T(\lambda) T(\mu), \quad \forall \lambda, \mu \in \Delta_{\theta} .
\end{gathered}
$$

Observación.- $S(t)$ es analítico sii $\exists K>0$ tal que $\|A S(t)\| \leq K t^{-1}, t>0$.

Teorema 1. (Lumer - Phillips)

Sea A un operador lineal con dominio denso $D(A)$ es un espacio de Hilbert $H$.

Si $A$ es disipativo y $\exists \lambda_{0}>0$ tales que $\operatorname{Im}\left(\lambda_{0} I-A\right)=H$, entonces $A$ es el generador infinitesimal de un semigrupo $C_{0}$ de contracciones en $H$.

\section{Corolario 1}

Sea $A$ es un operador lineal con dominio denso $D(A)$ en un espacio de Hilbert $H$.

Si $A$ es disipativo y $0 \in \rho(A)$ (el conjunto resolvente de $A$ ), entonces $A$ es el generador infinitesimal de un semigrupo $C_{0}$ de contracciones de $H$. 


\section{SOBRE LA ANALITICIDAD DEL SEMIGRUPO ...}

\section{Teorema 2.}

Sea $S(t)$ un semigrupo $C_{0}$ de contracción en un Espacio de Hilbert. Suponga que

$$
\rho(A) \supset\{i \beta, \beta \in \mathbb{R}\} \equiv i \mathbb{R} ;
$$

entonces $S(t)$ es analitica

$$
\Leftrightarrow \varlimsup_{|\beta| \rightarrow \infty}\left\|\beta(i \beta I-A)^{-1}\right\|<\infty
$$

acontece.

\section{Teorema 3.}

Sea $S(t)$ un $C_{0}$ semigrupo de contracciones en un Espacio de Hilbert. Entonces, $S(t)$ es exponencialmente estable

$$
\Leftrightarrow\left\{\rho(A) \supseteq i R, \varlimsup_{|\beta| \rightarrow \infty}\left\|(i \beta I-A)^{-1}\right\|<\infty\right\}
$$

\section{ANALITICIDAD DEL SEMIGRUPO ASOCIADO}

De la ecuación (1), haciendo $v=u_{t}$ obtenemos

$$
\left(\begin{array}{l}
u \\
v
\end{array}\right)_{t}=\left(\begin{array}{l}
u_{t} \\
v_{t}
\end{array}\right)=\left(\begin{array}{c}
u_{t} \\
u_{t t}
\end{array}\right)=\left(\begin{array}{c}
u_{t} \\
-\Delta^{2} u+\Delta u_{t}
\end{array}\right)
$$

entonces,

$$
\left(\begin{array}{l}
u \\
v
\end{array}\right)_{t}=\left(\begin{array}{cc}
0 & I \\
-\Delta^{2} & \Delta
\end{array}\right)\left(\begin{array}{l}
u \\
v
\end{array}\right)
$$

Definamos el Operador

$$
A:=\left(\begin{array}{cc}
0 & I \\
-\Delta^{2} & \Delta
\end{array}\right)
$$

(1) es equivalente a

$$
\mid \begin{aligned}
& Y_{t}=A Y \\
& Y(0)=\left(u_{0}, u_{1}\right)
\end{aligned}
$$

Construímos, la energía asociada al sistema. Multiplicando (1) por el $u_{t} \mathrm{e}$ integrando sobre $\Omega$ se tiene

$$
\int_{\Omega} u_{t t} \cdot u_{t}+\Delta^{2} u \cdot u_{t}-\Delta u_{t} \cdot u_{t}=0
$$


Usando la Identidad de Green, se obtiene

$$
\frac{1}{2} \frac{\partial}{\partial t} \int_{\Omega}\left|u_{t}\right|^{2}+\underbrace{\int_{\Omega} \Delta(\Delta u) u_{t}}_{J:=}+\int_{\Omega}\left|\nabla u_{t}\right|^{2}=0 .
$$

Pero

$$
\begin{aligned}
J & =-\int_{\Omega} \nabla(\Delta u) \nabla u_{t}+\underbrace{\int_{\Gamma} \frac{\partial \Delta u}{\partial v} \cdot u_{t}}_{=0} \\
& =\int_{\Omega} \Delta u \Delta u_{t}+\underbrace{\int_{\Gamma}^{\frac{\partial u_{t}}{\partial \nu}} \cdot \Delta u}_{=0}=\frac{1}{2} \frac{\partial}{\partial t} \int_{\Omega}|\Delta u|^{2} .
\end{aligned}
$$

Luego,

$$
\frac{\partial}{\partial t} \underbrace{\frac{1}{2}\left\{\int_{\Omega}\left|u_{t}\right|^{2}+|\Delta u|^{2} d x\right\}}_{E(t):=}=-\int_{\Omega}\left|\nabla u_{t}\right|^{2} d x \leq 0
$$

ie. $E(t) \downarrow, 0 \leq E(t) \leq E(0), \forall t>0$.

Ahora, consideremos

$$
X=H_{0}^{2}(\Omega) \times L^{2}(\Omega) \text { y } D(A)=\left(H_{0}^{2}(\Omega) \cap H_{\Delta}^{2} \cap H^{4}(\Omega)\right) \times H_{0}^{2}(\Omega)
$$

donde $H_{\Delta}^{2}=\left\{u \in H^{2}(\Omega)\right.$ talque $\left.\Delta u=0\right\}$.

Probaremos que el sistema (2) es disipativo.

$$
\begin{aligned}
D(A) \ni u,(A u, u) & =\left(\left(\begin{array}{l}
v \\
-\Delta^{2} u+\Delta v
\end{array}\right),\left(\begin{array}{l}
u \\
v
\end{array}\right)\right) \\
& =\int_{\Omega} \Delta v \Delta u+\int_{\Omega}-\left(\Delta^{2} u+\Delta v\right) v \\
& =\int_{\Omega} \Delta v \Delta u-\int_{\Omega} \Delta^{2} u v+\int_{\Omega} \Delta v \cdot v \\
& =\int_{\Omega} \Delta v \Delta u+\int_{\Omega} \nabla(\Delta u) \nabla v-\int_{\Omega}|\nabla v|^{2}+\int_{\Gamma} \frac{\partial \Delta u}{\partial v} \cdot v \\
& =\int_{\Omega} \Delta v \Delta u-\int_{\Omega} \Delta u \Delta v-\int_{\Omega}|\nabla v|^{2} \\
& =-\int_{\Omega}|\nabla v|^{2} d x \leq 0 .
\end{aligned}
$$

Por otro lado, necesitamos probar que $0 \in \rho(A)$. En verdad probaremos que $\exists(-A)^{-1} \in \mathcal{L}(X)$. 
Probaremos que $-A$ es inyectiva:

$$
\begin{aligned}
& -A U=0, \text { con } U=\left(\begin{array}{l}
u \\
v
\end{array}\right) \\
& -\left(\begin{array}{l}
v \\
-\Delta^{2} u+\Delta v
\end{array}\right)=\left(\begin{array}{l}
0 \\
0
\end{array}\right) \Rightarrow\left\{\begin{array}{c}
v=0 \\
-\Delta^{2} u=0
\end{array}\right. \\
& 0=-\int_{\Omega} \Delta^{2} u \cdot u=-\int_{\Omega} \Delta(\Delta u) u=-\int_{\Omega}|\Delta u|^{2}
\end{aligned}
$$

entonces $\|u\|=0 \Rightarrow u=0$. Así,

$$
U=\left(\begin{array}{l}
u \\
v
\end{array}\right)=\left(\begin{array}{l}
0 \\
0
\end{array}\right)
$$

La sobreyectividad resulta del Teorema de Lax-Milgram y la regularidad elíptica

Por lo tanto, el Corolario 1 nos permite concluir que $A$ es el generador infinitesimal de $T(t)$ un semigrupo $\mathrm{C}_{0}$ de contracción,

$$
U(t):=T(t) U_{0} \quad, \quad U_{0}=\left(\begin{array}{l}
u_{0} \\
u_{1}
\end{array}\right) ; U_{0} \in D(A),
$$

donde $U$ es la solución de $\left\{\begin{array}{l}x^{\prime}=A x \\ x(0)=U_{0}\end{array}\right.$.

En lo que sigue, probaremos que $T(t)$ es analítico, usando el Teorema 2.

Afirmación: $i \beta \subset \rho(A)$

Supongamos que exista $\beta$ tales que $i \beta \in \sigma(A)=\sigma_{p}(A)$. Luego, $\exists w \neq 0$ tal que

$$
A w=i \beta w .
$$

Denotemos

$$
w=\left(\begin{array}{l}
u \\
v
\end{array}\right)
$$

Así (3) queda expresado por

$$
\left(\begin{array}{c}
v \\
-\Delta^{2} u+\Delta v
\end{array}\right)=i \beta\left(\begin{array}{l}
u \\
v
\end{array}\right)
$$

es decir,

$$
\mid \begin{aligned}
& v=i \beta u \\
& -\Delta^{2} u+\Delta v=i \beta v
\end{aligned}
$$


Sustituyendo (4a) en (4b) obtenemos

$$
-\Delta^{2} u+i \beta \quad \Delta u=(i \beta)^{2} u=-\beta^{2} u .
$$

Multiplicando (5) por $u$ e integrando obtenemos

$$
-\int_{\Omega}|\Delta u|^{2} d x-i \beta \int_{\Omega}|\nabla u|^{2} d x=-\beta^{2} \int_{\Omega}|u|^{2} d x .
$$

Por otro lado,

$$
(A w, \bar{w})=(i \beta w, \bar{w})=i \beta(w, \bar{w})=i \beta\|w\|^{2} .
$$

Tomando la parte real, conseguimos

$$
\operatorname{Re}(A w, \bar{w})=0 .
$$

De (7) se obtiene

$$
\begin{aligned}
\left(\left(\begin{array}{c}
v \\
-\Delta^{2} u+\Delta u
\end{array}\right),\left(\begin{array}{l}
\bar{u} \\
\bar{v}
\end{array}\right)\right) & =\int_{\Omega} \Delta v \cdot \overline{\Delta u}+\int_{\Omega}\left(-\Delta^{2} u+\Delta v\right) \bar{v} \\
& =\int_{\Omega} \Delta v \cdot \overline{\Delta u}-\int_{\Omega} \Delta u \Delta \bar{v}-\int_{\Omega}|\nabla u|^{2} \\
& =2 i \operatorname{Im}\left\{\int_{\Omega} \Delta v \overline{\Delta u}\right\}-\int_{\Omega}|\nabla u|^{2}
\end{aligned}
$$

(8) y (9) nos conducen a

$$
\begin{gathered}
-\int_{\Omega}|\nabla v|^{2}=0 \\
\text { i.e } \quad v=0 \text { en } H_{0}^{1} \Rightarrow v=0 \text { en } L^{2} \Rightarrow u=0 \text { en } L^{2} \\
\downarrow \\
u=0 \text { en } H_{0}^{1}
\end{gathered}
$$

de (6) y (10) conseguimos

$$
\begin{aligned}
& -\int_{\Omega}|\Delta u|^{2} d x-i \beta \underbrace{\int_{\Omega}|\nabla u|^{2} d x}_{=0}=-\beta^{2} \underbrace{\int^{|u|^{2} d x}}_{=0} \\
& \therefore \int_{\Omega}|\Delta u|^{2} d x=0 \\
& \therefore\|w\|_{X}=\int_{\Omega}|\Delta u|^{2} d x+\int_{\Omega}|v|^{2} d x=0 \Rightarrow w=0(\Rightarrow \Leftarrow) .
\end{aligned}
$$

Sea

$$
(i \beta I-A) U=F \quad, \quad U=\left(\begin{array}{l}
u \\
v
\end{array}\right) \in D(A), F=\left(\begin{array}{l}
F_{1} \\
F_{2}
\end{array}\right)
$$


Probamos que $|\beta|\|R(i \beta, A) F\| \leq C|F|_{X}$

$$
(a) \longleftrightarrow \mid \begin{aligned}
& i \beta u-v=F_{1} \\
& i \beta v+\Delta^{2} u-\Delta v=F_{2}
\end{aligned}
$$

Sustituyendo (11) en (12), obtenemos

$$
\begin{aligned}
& i \beta\left\{i \beta u-F_{1}\right\}+\Delta^{2} u-\Delta\left(i \beta u-F_{1}\right)=F_{2} \\
& -\beta^{2} u-i \beta F_{1}+\Delta^{2} u-i \beta \Delta u+\Delta F_{1}=F_{2} \\
& -\beta^{2} u+\Delta^{2} u-i \beta \Delta u=F_{2}-\Delta F_{1}+i \beta F_{1} .
\end{aligned}
$$

Multiplicando (13) por $\overline{\Delta u}$, obtenemos

$$
-\beta^{2} u \overline{\Delta u}+\Delta^{2} u \overline{\Delta u}-i \beta \Delta u \overline{\Delta u}=\left(F_{2}-\Delta F_{1}\right) \Delta \bar{u}+i \beta F_{1} \Delta \bar{u} .
$$

Integrando sobre $\Omega$ y usando la Identidad de Greeṇ obtenemos

$$
\begin{aligned}
& \beta^{2} \int_{\Omega}|\nabla u|^{2} d x-\int_{\Omega}|\nabla(\Delta u)|^{2} d x+\int_{\Gamma} \frac{\partial}{\partial \nu}(\Delta u) \overline{\Delta u}-i \beta \int_{\Omega}|\Delta u|^{2}= \\
& =\int_{\Omega}\left(F_{2}-\Delta F_{1}\right) \Delta \bar{u}+i \beta \int_{\Omega} F_{1} \Delta \bar{u} .
\end{aligned}
$$

Tomando conjugado a igualdad (11), obtenemos

$$
\begin{aligned}
i \beta \int_{\Omega} F_{1} \Delta \bar{u} d x & =i \beta \int_{\Omega} \Delta F_{1} \bar{u}=\int_{\Omega} \Delta F_{1} \cdot i \beta \bar{u}=\int_{\Omega} \Delta F_{1}\left(-\bar{v}-\overline{F_{1}}\right) \\
& =\int_{\Omega} \Delta F_{1} \bar{v}-\int_{\Omega} \Delta F_{1} \cdot \overline{F_{1}}=-\int_{\Omega} \Delta F_{1} \bar{v}+\int_{\Omega}\left|\nabla F_{1}\right|^{2} .
\end{aligned}
$$

Sustituyendo (15) en (14) y tomando la parte imaginaria, obtenemos

$$
\begin{aligned}
-\beta \int_{\Omega}|\Delta u|^{2} d x & =\operatorname{Im}\left\{\int_{\Omega}\left(F_{2}-\Delta F_{1}\right) \Delta \bar{u}\right\}-\operatorname{Im} \int_{\Omega} \Delta F_{1} \bar{v} \\
& =\operatorname{Im}\left\{\int_{\Omega}\left(F_{2}-\Delta F_{1}\right) \Delta \bar{u}-\int_{\Omega} \Delta F_{1} \bar{v}\right\} .
\end{aligned}
$$

Multiplicando la ecuación (12) por $\bar{v}$ e integrando sobre $\Omega$, obtenemos

$$
i \beta \int_{\Omega}|v|^{2}+\int_{\Omega} \Delta^{2} u \bar{v}-\int_{\Omega} \Delta v \bar{v}=\int_{\Omega} F_{2} \bar{v}
$$

Utilizando la Identidad de Green

$$
i \beta \int_{\Omega}|v|^{2}+\int_{\Omega} \Delta u \Delta \bar{v}+\int_{\Omega}|\Delta v|^{2}=\int_{\Omega} F_{2} \bar{v}
$$


Tomando el Laplaciano a la ecuación (11) tenemos,

$$
i \beta \Delta u-\Delta v=\Delta F_{1}
$$

Multiplicando la ecuación (18) por $\Delta \bar{u}$ e integrando sobre $\Omega$ tenemos,

$$
i \beta \int_{\Omega}|\Delta u|^{2} d x-\int_{\Omega} \Delta u \Delta \bar{u} d x=\int_{\Omega} \Delta F_{1} \Delta \bar{u} d x
$$

Sumando (17) y (19) obtenemos,

$$
\begin{aligned}
& \int_{\Omega}|\nabla u|^{2} d x+i \beta\left\{\int_{\Omega}|v|^{2}+|\Delta u|^{2} d x\right\}+2 i \operatorname{Im}\left\{\int_{\Omega} \Delta u \Delta \bar{v}\right\} \\
& =\int_{\Omega} F_{2} \bar{v}+\int_{\Omega} \Delta F_{1} \Delta \bar{u} .
\end{aligned}
$$

Pero, se observa que

$$
\begin{aligned}
\int_{\Omega} \Delta u \Delta \bar{v} & =\int_{\Omega} \Delta u\left\{i \beta \Delta \bar{u}-\Delta \bar{F}_{1}\right\} \\
& =i \beta \int_{\Omega}|\Delta u|^{2} d x-\int_{\Omega} \Delta u \Delta \bar{F}_{1} .
\end{aligned}
$$

Luego,

$$
\operatorname{Im} \int_{\Omega} \Delta u \Delta \bar{v}=-\beta \int_{\Omega}|\Delta u|^{2} d x-\operatorname{Im} \int_{\Omega} \Delta u \Delta \overline{F_{1}} .
$$

Sustituyendo (21) en (20) obtenemos,

$$
\begin{aligned}
& \int_{\Omega}|v|^{2} d x+i \beta\left\{\int_{\Omega}|v|^{2}+|\Delta u|^{2} d x\right\}-2 i \beta \int_{\Omega}|\Delta u|^{2} d x \\
& -2 i \operatorname{Im} \int_{\Omega} \Delta u \Delta \overline{F_{1}}=\int_{\Omega} F_{2} \bar{v}+\int_{\Omega} \Delta F_{1} \Delta \bar{u} .
\end{aligned}
$$

Tomando la parte imaginaria, obtenemos

$$
\beta\left\{\int_{\Omega}|v|^{2}-|\Delta u|^{2} d x\right\}=2 \operatorname{Im} \int_{\Omega} \Delta u \Delta \overline{F_{1}}+\operatorname{Im}\left\{\int_{\Omega} F_{2} \bar{v}+\int_{\Omega} \Delta F_{1} \Delta \bar{u}\right\} .
$$

sumando las ecuaciones (22) $y-2(16)$ tenemos

$$
\begin{aligned}
\beta \int_{\Omega}|v|^{2}-|\Delta u|^{2} d x= & \operatorname{Im}\left\{\int_{\Omega} 2 \Delta u \Delta F_{1}+F_{2} \bar{v}+\Delta F_{1} \Delta \bar{u}\right\} \\
& -2 \operatorname{Im}\left\{\int_{\Omega}\left(F_{2}-\Delta F_{1}\right) \Delta \bar{u}-\Delta F_{1} \bar{v}\right\}
\end{aligned}
$$

tomando $|$.$| se consigue,$ 


$$
\begin{aligned}
& |\beta| \underbrace{\int_{\Omega}\left\{|v|^{2}+|\Delta u|^{2}\right\}}_{|U|_{X}^{2}} d x \leq|F|_{X}|U|_{X} \\
& |\beta||U|_{X}^{2} \leq C|F|_{X}|U|_{X} \\
& |\beta||U|_{X} \leq C|F|_{X} \\
& |\beta|\|R(i \beta, A) F\| \leq C|F|_{X} \\
& |\beta|\|R(i \beta, A)\| \leq C .
\end{aligned}
$$

Tomando el límite cuando $|\beta| \rightarrow+\infty$, se tiene $\lim _{|\beta| \rightarrow+\infty}|\beta|\|R(i \beta, A)\| \leq C$, donde $C$ es una constante positiva.

\section{REFERENCIAS BIBLIOGRÁFICAS}

[1] Adams, R. A. Solobev Spaces. Academic Press, New York. (1975).

[2] Liu-Zheng. Semigroups associated with dissipative systems. Chapman-Hall/ CRC. (1999).

[3] Pazy, A. Semigroups of Linear Operators and applications to partial Differential Equations. Springer, New York. (1983).

[4] Prüss, J. On the spectrum of $C_{0}$ Semigroups. Trans AMS 284, pag. 847-857. (1984).

[5] Renardy, M. Hrusa, W. J. and Nohel, J.A. Mathematical problems in Viscoelasticity. Pitman monographs and surveys in Pure and Applied Mathematics 35, John Wiley \& Sons. New York. (1987).

[6] Wyler, A. Stability of wave equations with dissipative boundary conditions in a bounded domain. Differential and Integral Equations, Vol 7, number 2, 345 336. (1994). 